\title{
Based on SWA Sensor Energy Consumption Under the Sports Fitness and Strength of the Correlation Research
}

\author{
Zhenyu Tang ${ }^{*}, 1$ and Wei Yang ${ }^{2}$ \\ ${ }^{I}$ Department of Physical Education, Heilongjiang Bayi Agricultural University, Daqing, Heilongjiang, China \\ ${ }^{2}$ Department of Physical Education, Dalian University of Technology, Dalian, Liaoning, China
}

\begin{abstract}
SWA sensor is used to measure the treadmill walking running and cycling on the spot when the energy consumption, and compared with the indirect method of measuring thermal measurement results, the SWA to measure the effectiveness of the energy consumption for fitness, provide a reference for accurate application of the sensor. In 15 adult male in tablet exercise treadmill to complete the $4.8 \mathrm{~km} / \mathrm{h}, 6.4 \mathrm{~km} / \mathrm{h}, 8.0 \mathrm{~km} / \mathrm{h}$ speed of three movement walk, run, and complete $10 \mathrm{~km} / \mathrm{h}$ in indoor, $13 \mathrm{~km} / \mathrm{L}, 15 \mathrm{~km} / \mathrm{h}$ cycling sport, using SWA (two software version V6.1, V7.0) and indirect method of measuring thermal forecast movement of energy consumption, to predict data matching test, regression analysis, correlation analysis, Ie coefficient and consistency check. Results: (1) gWA6.1 overestimated the treadmill run go ( $1 \%$ overvalued) and field cycling of energy consumption ( $9 \%$ overvalued), but has good correlation and consistency ( $\mathrm{r}$ $=0.91$, the ICC $=0.91)$. Gara sensor $(2)$ prediction of energy consumption for fitness value is far greater than the indirect method of measuring thermal prediction of energy consumption, and to predict the energy consumption difference increased with the increase of speed increasing, and the sensor old version overestimate low intensity exercise energy consumption and high intensity of energy consumption is different, but the estimate of the energy consumption and had a higher correlation.
\end{abstract}

Keywords: Energy consumption, fitness, indirect calorimetric, multifunction sensor, validity.

\section{INTRODUCTION}

Exercise is an important content of daily physical activity, measuring its energy consumption, not only can provide the basis for evaluating dynamic lifestyle, but also help the body energy balance management to complete the quantitative targets for fitness. Indirect calorimetric method [1] and double standard Water [2] has been confirmed as the gold standard for measuring energy consumption, but due to high cost, complex operation, it is difficult to used to measure large samples free life state of energy consumption. At present, the energy consumption measurement instruments and tools tend to develop in the direction of simple and effective. Physical activity $\log$ is a common method in the investigation and study, but the existing studies have shown that the result and the actual energy consumption, the deflection and consistency. Sensor technology has been widely used in the human body physical activity measurement of energy consumption, to learn every day of physical activity level and energy consumption to provide objective data [3].

Motion sensor is a kind of mechanical or electronic device that can be fixed on the body induction to the movement condition of the body or body, body displacement and record the data, by the mathematical model of internal conversion into the daily energy consumption value. Motion sensors: nitrogen is small, easy to wear, impact on the

*Address correspondence to this author at the Department of Physical Education, Heilongjiang Bayi Agricultural University, Daqing, Heilongjiang, China; Tel: +86-13039878781; E-mail: Fswxwhy@163.com everyday life is small, compared with physical activity recording accuracy have greatly improve, become in recent years commonly used measuring tools of energy consumption of free life condition [4].

Sense Wear Armband Pr03 [5] is the Body of Media production belt type multifunctional sensor, an arm can dynamically test subjects related data of daily physical activity and sleep behavior. SWA when measuring energy consumption, its data from five built-in sensors, dual-axis accelerometer, respectively, two skin conductance response sensor, skin temperature sensor, and heat flux sensor. Compared with other acceleration sensor meter, heat flux sensor is one of the unique technology of SWA, measures and the heat loss with acceleration sensor data, compared with the single axis or multi-axis accelerometer, can more accurately predict the body energy consumption values every day. In addition, given the general state of acceleration sensor is difficult to separate a sit still and very mild physical activity, and SWA can detect low intensity exercise the skin temperature of reaction and electricity changes, therefore, for predicting energy consumption low intensity exercise has more advantages. SWA has been widely used in the past ten years [6]. SWA is used to measure the free life of energy consumption, compared with DLW measurement is undervalued, but two measurement methods in measuring the consistency of the daily energy consumption when is better. But there are also studies pointed out that the only subjects daily energy consumption high, SWA will underestimate energy consumption, and low energy consumption every day, SWA exists overestimate the phenomenon of energy consumption, likewise, measuring 
the consistency of both were in an acceptable range [7]. SWA is used to measure the movement of energy consumption, various versions of SWA instrument test data, and the same instrument testing data after different versions of the software to calculate the energy consumption value is also different. SWA (Pro, V3.2) underestimated the most movement of energy consumption, have overestimated the upper arm is given priority to the movement of energy consumption. Compared with the indirect method of measuring heat, in software version V3.9 and V4.1, use the formula to predict energy consumption results improve. Some researchers use the same as this research instrument and software version of SWA (Pr03, V6.1), in the prediction of low intensity exercise overestimated the energy consumption.

The above research results, SWA for measurement of daily physical activity and exercise energy consumption are not the same, whether the used to measure the effectiveness of the energy consumption for fitness, is unknown. This study of 15 adult male as the research object, SWA sensor is used to measure the treadmill go running and cycling on the spot when the energy consumption, and compared with the indirect method of measuring thermal measurement results, the SWA to measure the effectiveness of the energy consumption for fitness, provide a reference for accurate application of the sensor.

\section{RESEARCH OBJECTS AND METHODS}

15 the object of study for adult men, with an average age of 23 years old, the youngest is 20 years old, so much age 26 (Table 1). Subjects by history and physical examination, no respiratory system, cardiovascular system, endocrine system disease, exercise habits are greater than $6 \mathrm{~h}$ per week of moderate intensity exercise.

\subsection{Research Method}

All the test content of the test scheme, participants of discontinuous day to finish within a week. To exercise treadmill testing, complete bicycle test in a week. Order two tests are completed at random, each test Wear the Sense Wear Pro Armband (SWA), at the same time the use of portable gas metabolism instrument complete indirect measurements of thermal energy consumption test. Test before $12 \mathrm{~h}$ bland diet, no violent activity, not smoking and drinking caffeinated beverages. When test subjects wearing comfortable cotton sports clothes and sports shoes, on an empty stomach after 2 to $3 \mathrm{~h}$.

Exercise treadmill test, the test subjects care plate movement run on adaptive movement before $3 \mathrm{~min}$. Instructed participants hold the handrail after beginning of the test stand on the plate movement run $5 \mathrm{~min}$, complete quiet condition data collection, and then complete each 4.8 $\mathrm{km} / \mathrm{h}$ (3 MPH), $6.4 \mathrm{~km} / \mathrm{h}$ (4 MPH), $8.0 \mathrm{~km} / \mathrm{h}$ (5 MPH) 3 speed of walk, run, slope is set to $0,6 \mathrm{~min}$ each speed, movement in the process of the subjects don't hold handrail, a total of 18 min continuous movement. Exercise treadmill testing laboratory temperature $1.5{ }^{\circ} \mathrm{C}, 21.7{ }^{\circ} \mathrm{C}$ soil test environment Q concentration was $23.93 \%$, CQ concentration was $0.03 \%$.

Indoor cycling test, bicycle test in a 90 × $50 \mathrm{~m}$ indoor empty workshop, floor for concrete, told participants ride around in circles. Before the test, according to the figure of each subject condition adjustment of bicycle saddle, handlebar and pedal position, in order to achieve the body slightly forward, arms slightly bent the best cycling posture. Subjects to ride a bicycle to fitness movement $3 \mathrm{~min}$. Test after the start of each subject to keep the seat first quiet state of five $1 \mathrm{~min}$, then each person according to the bicycle on the clock display speed of $10 \mathrm{~km} / \mathrm{h}, 13 \mathrm{~km} / \mathrm{h}$ and $15 \mathrm{~km} / \mathrm{h}$ speed of three cycling, the rest every 2 speed between 1-3 min, the rest to the quiet heart rate shall prevail. Subjects are conducted using the same bike test, for ordinary light car, bicycle tires is 26 inches in diameter, tires inflated.

Test equipment, all needs to connect the computer software input before each test participants' personal information (date of birth, height, weight, sex, dominant hand information, whether smoking), then $5 \mathrm{~min}$ in advance to wear in the subjects of the dominant hand side of the upper arm triceps, keep the upper arm skin is dry before wearing. Cable should be used in each participant's test after the instrument is connected to the computer, through Sensewear 6.1 software will guide person computer and save the data for the software can identify the SWD file, and then on to the next participant's test. Tests using the same heart rate table (Polar, Finland) and indirect measurement instruments. Height measurement using xin donghua teng CJ - II (automatic) height tester, weight with high precision digital display electronic body scale RCS - 160; Body fat content using GE dual-energy X-ray absorptiometry scanner.

\subsection{Data Handling}

Use Sense Wear 6.1 and Sense Wear two software version 7.0 of Sense Wear Pro Armband Pr03 measured by SWD file data calculation, read quiet state, participants go run and bike every average speed of the whole data. The absolute value of energy consumption, according to the software to calculate the energy consumption of per kilogram of body weight. With $3 \mathrm{~b}$ gas metabolism instrument equipped with software Meta - Soft 3.9 to collect data for processing, energy consumption, relative indexes of energy consumption, the test shows that the index of $20 \mathrm{~s}$ time interval mean, read quiet state, participants go run and bike every speed the way the average of the data, as shown in Table 2.

Table 1. Research objects general information.

\begin{tabular}{|c|c|c|c|c|c|}
\hline $\mathbf{N}$ & Age (Years Old) & Height (cm) & Weight (kg) & Weight Index BMI & Body Fat Rate (\%) \\
\hline \hline 15 & $23 \pm 3$ & $174.2 \pm 5.1$ & $68.3 \pm 11$ & $22.5 \pm 3.4$ & $17 \pm 9.4$ \\
\hline
\end{tabular}


Table 2. Research energy consumption in fitness.

\begin{tabular}{|c|c|c|c|c|}
\hline & Test State & SWA7.0 & SWA6.1 & IC \\
\hline \hline \multirow{5}{*}{ Walking running } & stand quiet & $5.81 \pm 0.71$ & $1.39 \pm 0.17$ & $1.75 \pm 0.28$ \\
\cline { 2 - 5 } & $4.8 \mathrm{~km} / \mathrm{h}$ & $22.06 \pm 4.03$ & $5.26 \pm 0.96$ & $4.85 \pm 0.75$ \\
\cline { 2 - 5 } & $6.4 \mathrm{~km} / \mathrm{h}$ & $30.21 \pm 6.5$ & $7.18 \pm 1.53$ & $6.97 \pm 1.00$ \\
\cline { 2 - 5 } & $8.0 \mathrm{~km} / \mathrm{h}$ & $41.52 \pm 7.38$ & $9.91 \pm 1.76$ & $10.23 \pm 1.33$ \\
\cline { 2 - 5 } & average value of 3 speeds & $31.26 \pm 5.43$ & $7.45 \pm 1.29$ & $7.35 \pm 0.98$ \\
\cline { 2 - 5 } & Sit quiet & $5.26 \pm 0.71$ & $1.26 \pm 0.17$ & $1.90 \pm 0.34$ \\
\cline { 2 - 5 } & $10 \mathrm{~km} / \mathrm{h}$ & $19.99 \pm 7.56$ & $4.78 \pm 1.81$ & $4.04 \pm 0.94$ \\
\cline { 2 - 5 } & $13 \mathrm{~km} / \mathrm{h}$ & $25.88 \pm 8.30$ & $6.19 \pm 1.98$ & $5.31 \pm 0.81$ \\
\cline { 2 - 5 } & $15 \mathrm{~km} / \mathrm{h}$ & $25.76 \pm 8.60$ & $6.164 \pm 2.05$ & $6.32 \pm 0.99$ \\
\cline { 2 - 5 } & average value of 3 speeds & $23.88 \pm 6.65$ & $5.71 \pm 1.59$ & \multicolumn{2}{c|}{$5.22 \pm 0.88$} \\
\hline
\end{tabular}

In the resting state energy consumption predicting, SWA7.0 comparing with IC prediction value, overestimates $232 \%$ ( stand quiet state) and $177 \%$ ( sit quiet state) energy consumption. In walking running and cycling, the difference between SWA7.0 and IC predicted energy consumption constant increase as sports speed increasing (overestimate $306 \%-395 \%$ ) and that difference in walling running is bigger than that in cycling between SWA7.0 and IC.

In the resting state energy consumption prediction SWA6.1 underestimates 20\%(stand quiet state) and 34\%( sit quiet state) energy consumption; When prediction walking running and cycling, it has the same difference tendency as IC, both overestimate sports energy consumption in first two speed( walking running is $9 \%$ and $3 \%$, cycling is $18 \%$ and $17 \%$ ) and underestimate sports energy consumption at the 3rd speed( walking running is $3 \%$, cycling is $4 \%$ ). The difference in predicting walking running energy consumption between SWA6.1 and IC ( average value is o.3 $\mathrm{kcal} / \mathrm{min}$, overestimate $1 \%$ ) less than that of cycling predicting ( average value is $1.45 \mathrm{kcal} / \mathrm{min}$, overestimate $9 \%)$.

\section{ANALYSIS AND DISCUSSION}

Go run and bike is a common exercise way. This study chooses the normal speed $(4.8 \mathrm{~km} / \mathrm{h})$, quick walk $(6.4 \mathrm{~km} / \mathrm{h})$, jogging $(8.0 \mathrm{~km} / \mathrm{h})$ the three commonly used go running speed and slow cycling $(10 \mathrm{~km} / \mathrm{h})$, medium speed bike (13 $\mathrm{km} / \mathrm{h})$, rapid cycling $(15 \mathrm{~km} / \mathrm{h})$ the three commonly used bike speed, partly reflects a common form of exercise. When using SWA to go running and cycling measure energy consumption, can reflect the characteristics of energy consumption of when measuring exercise.

Quiet condition of energy consumption represents the state of the body's energy metabolism of cells, for most of the daily total energy consumption of content, its forecasting accuracy directly affect the accuracy of the daily total energy consumption. SWA7. O and SWA6.1 in measuring position and seat quiet state energy consumption compared with
IC show very significant difference. Although SWA7. O overestimated the quiet state of energy consumption $(232 \%$ and $177 \%$ ), whereas SWA6.1 underestimated the quiet state of energy consumption $(20 \%$ and $34 \%)$, but the difference between the IC and correlation and consistency is good. The results measured in SWA results of energy consumption of the deflection, and the existing research reports in larger, on the one hand, the reason may be that the instruments of both research and version, there is a more important reason may be related to the participants before the formal into the quiet state test SWA wear time is too short, only $5 \mathrm{~min}$ ). Referred to in the study of the subjects in the first $10 \mathrm{~min}$ data differences, may SWA each sensor before the test needs a long time to adapt in the work.

SWA6.1 in predicting go running and biking with no difference between the IC prediction, energy consumption and good correlation and consistency, can effectively predict both energy consumption, and SWA 6.1 prediction go running energy consumption correlation ( $\mathrm{r}$ ) and consistency were superior to predict energy consumption in the sport. SWA 6.1 in normal walking speed and fast measurement, overestimated $0.210 .42 \mathrm{kcal} / \mathrm{min}(\mathrm{r}=0.79,0.81)$ of energy consumption. But with the increase of go running speed, SWA6.1 underestimated the jogging $0.33 \mathrm{kcal} /$ rain of energy consumption (3\%), and according to the existing research results, in the prediction of high speed running (10 preceding $\sim 15.84 \mathrm{~km} / \mathrm{h}$ ), underestimate the trend of energy consumption increase. That with the increase of speed, SWA 6.1 to underestimate energy consumption may be linked to the cause of most of subjects in along with the increase of the intensity have sweat phenomenon, the skin sweat has affected the SWA built-in heat flux sensor measurements, then affect the accuracy of the SWA prediction formula. SWA 6.1 in the prediction of cycling, although the overall energy consumption $9 \%$ overvalued, but no statistical differences compared with IC data, the main reason is the difference between power cycling and field white crane. SWA predict motion energy consumption is mainly dependent on the built-in acceleration sensor, and this kind 
of sensor in the prediction of movement need not bear its weight, such as cycling, tend to underestimate its energy consumption, especially in power cycling, don't need to control their own body weight impact of balance and make an extra effort, therefore, SWA underestimate the possibility of increased. In this study field bicycle test indoor capacious space use, close to the actual state of cycling, there may be some additional participants body movement to keep the balance when riding, caused the SWA6.1 when compared with IC overestimated the cycling of energy consumption.

At present, SWA versions of the software has been updated to V7. O, there is no doubt that the prediction formula of energy consumption has been updated, but exactly how to predict the effectiveness of the energy consumption, there is no study confirmed. Mentioned in Soric and so on in the study, the researchers used V7. O with V6.1 data shows that the energy consumption data can be, but not to both data for statistical analysis. SWA7.0 as Body Media company's new software version, there is no research shows that the validity and reliability of the predicted energy consumption data. . But through this study found that although SWA7 o solve the previous version to underestimate the movement's energy consumption problem, but its forecast of energy consumption is greater than that of IC to predict energy consumption (differential value of 3.36 $\sim 31.29 \mathrm{kcal} / \mathrm{min}$, overestimate $177 \% \sim 395 \%$ ). The present data show that SWA7.0 associated with SWA 6.1 data are almost completely. In addition, SWA7.0 in predicting go running and cycling when its energy consumption and IC difference increased with the increase of speed increasing, this performance is not consistent with SWA6.1, may change significantly with manufacturer predicts the energy consumption. But in running energy consumption prediction walked through SWA7.0 correlation and consistency is still superior to predict bicycle exercise energy consumption), presumably, although the manufacturer change significantly predicts the energy consumption of the formula, but I could only change the certain numerical value, and the calculation principle and mode does not change the larger.

Existing studies have shown that SWA when measuring total daily energy consumption is superior to other accelerometer, and compared with other accelerometer can more accurately predict low intensity exercise energy consumption, the main reason lies in the accelerometer is imperceptible to sit motionless state and very mild physical activity, therefore, may underestimate the power consumption of a very mild physical activity. SWA7.0 and SWA 6.1 in this study are in low intensity exercise overestimated the movement of energy consumption, reflects the multiple sensors in energy consumption prediction formula plays a role. But as a result of SWA manufacturer has not released its energy consumption prediction formula, therefore, it is difficult to find the SWA in predicting energy consumption compared with IC data appear overvalued or undervalued reasons and improving methods. But not according to the energy consumption of SWA6.1 forecasting with bicycle exercise intensity increases with the increase of phenomenon, presumably SWA to predict energy consumption level of the sports from the accelerometer data, and the measurement function of added speed meter to the cycling with the small amplitude motion of the upper extremity is restricted, as a result, the same physical activity and it is difficult to measure the movement under load changes the body's power, and lead to predict energy consumption differences. Future SWA formula can consider adding other sensors, such as heat flux sensor data of the weights. This problem has been in the formula of SWA7.0 improved better.

In addition, due to the limitation of conditions in this study have two limitations. First, participants choose 15 adult male, and the normal weight (BMI 22.5 soil 3.4), but the results can be applied in other ages, overweight and obese people, and even the crowd of different physique level, needs to be confirmed by further research. SWA, for example, energy consumption prediction formula including heat flux sensor data, and because overweight and obese people will wear instrument in the subcutaneous fat layer is thick and impact energy consumption forecast accuracy, is currently unknown. Second, this study only chose to run go running and cycling on the spot as the main way of exercise, but if SWA can accurately predict the other way when the exercise energy expenditure, or greater strength, longer duration of movement and movement under different environmental conditions, also need to be solved in future studies.

\section{CONCLUSION}

SWA sensor prediction of energy consumption for fitness value is far greater than the indirect method of measuring thermal prediction of energy consumption, and to predict the energy consumption difference increased with the increase of speed increasing, and the sensor old version overestimate low intensity exercise energy consumption and high intensity of energy consumption is different, but the estimate of the energy consumption and had a higher correlation between indirect method of measuring heat and consistency.

\section{CONFLICT OF INTEREST}

The authors confirm that this article content has no conflict of interest.

\section{ACKNOWLEDGEMENTS}

This work is supported by 2014 year Heilongjiang Province project of social sciences research; No: 14B046.

\section{REFERENCES}

[1] P. Chen, Z. Qin, Y. Yin, and C. Xia, "Correlation analysis of current situation of regional athletics sports development and society population structure in China," Journal of Beijing Sport University, vol. 30, no. 12, pp. 1610- 1613, 2007.

[2] H. Guo, "Summary on Chinese sports population research since 1980s," China Sport Science and Technology, vol. 43, no. 3, pp. 36-40, 2007. 
[3] H. Li, H. Xue, and W. Feng, "Sociological analysis of comparison of chinese population with the sports population of Chinese," Journal of Xi'an Institute of Physical Education, vol. 24, no. 4, pp. 25-28, 2007.

[4] L. Li, J. Yang, T. Yang, and L. Xu, "A Research on the sustainable development of evaluation index system of regional sports industry," Journal of Beijing Sport University, vol. 18, no. 9, pp. 33-40, 2010.

[5] Z. Miao, and C. Qin, "Sociological analysis of contemporary Chinese sports population structures," Journal of Physical Education, vol. 13, no. 1, pp. 119-121, 2006.
[6] J. Xue, and J. Wang, "Analysis on development level of competitive sports of Eastern China in the "eleventh five-year" period," Bulletin of Sport Science \& Technology, vol. 19, no. 4, pp. 57-59, 2011.

[7] D. Yan, "Development of sports for all under circumstance of building well-off society," Journal of Wuhan Institute of Physical Education, vol. 40, no. 1, pp. 15-19, 2006.

Received: September 16, 2014

Revised: December 23, 2014

Accepted: December 31, 2014

(C) Tang and Yang; Licensee Bentham Open.

This is an open access article licensed under the terms of the (https://creativecommons.org/licenses/by/4.0/legalcode), which permits unrestricted, non-commercial use, distribution and reproduction in any medium, provided the work is properly cited. 